\title{
BOUNDARY VALUE PROBLEM FOR FUCHSIAN PARTIAL DIFFERENTIAL EQUATIONS AND REFLECTION OF SINGULARITIES
}

\author{
TOSHINORI ÔAKU \\ Department of Mathematics, Yokohama City University \\ 22-2 Seto, Kanazawa-ku, Yokohama, 236 Japan
}

Introduction. We study linear partial differential equations of Fuchsian type with respect to a hypersurface in $\mathbb{R}^{n}$. The notion of Fuchsian equations was introduced by Baouendi-Goulaouic [1] and is almost equivalent to the notion of equations with regular singularities in the weak sense defined by KashiwaraOshima [2]. We note here that these notions have been generalized to systems and to submanifolds of arbitrary codimension by Oshima [10] and Laurent-Monteiro Fernandes [3].

First, we present a method for defining boundary values of hyperfunction solutions to a Fuchsian partial differential equation with respect to a hypersurface. For single partial differential equations with regular singularities (in the weak sense), there have been two methods to define boundary values of hyperfunction solutions: one is a very general but rather abstract method of Kashiwara-Oshima [2], and the other is a very concrete but rather restrictive (the characteristic exponents must be constant) method by Oshima [9]. Our method is close to the latter, but not restrictive.

Our main purpose is to study the reflection of singularities of hyperfunction solutions to a Fuchsian linear partial differential equation under some boundary condition. One of the most typical examples is the equation

$$
x_{1}\left(\frac{\partial^{2} u}{\partial x_{1}^{2}}-\frac{\partial^{2} u}{\partial x_{2}^{2}}\right)+a_{1}(x) \frac{\partial u}{\partial x_{1}}+a_{2}(x) \frac{\partial u}{\partial x_{2}}+b(x) u=0
$$

in $\mathbb{R}^{2} \ni x=\left(x_{1}, x_{2}\right)$, where $a_{1}, a_{2}, b$ are real-analytic functions and $u$ is a hyperfunction on $x_{1}>0$ satisfying a certain boundary condition on $x_{1}=0$. We give some sufficient conditions for the reflection of (analytic) singularities of $u$ at the boundary $x_{1}=0$. 
We can also treat, e.g., the following (rather special) equations:

$$
\frac{\partial^{2} u}{\partial x_{1}^{2}}-x_{1}^{k} \frac{\partial^{2} u}{\partial x_{2}^{2}}=0, \quad x_{1} \frac{\partial^{2} u}{\partial x_{1}^{2}}-x_{1}^{k} \frac{\partial^{2} u}{\partial x_{2}^{2}}+a_{1}\left(x_{2}\right) \frac{\partial u}{\partial x_{1}}=0
$$

with an integer $k \geq 0$ and a real-analytic function $a_{1}\left(x_{2}\right)$.

When the boundary is non-characteristic, such problems have been studied by Lax-Nirenberg [4] for the $C^{\infty}$-singularity and by Schapira [11] for the analytic singularity. But it seems that there have been no results for degenerate equations including the above three examples.

1. Boundary values of hyperfunction solutions of Fuchsian partial differential equations

1.1. Fuchsian partial differential equations and their formal elementary solutions following Tahara. First let us recall the notion of Fuchsian operators with respect to a hypersurface in accordance with [1]. Let $x=\left(x_{1}, x_{2}, \ldots, x_{n}\right)$ be the coordinate of the $n$-dimensional real Euclidean space $M=\mathbb{R}^{n}$. We also write $x=\left(x_{1}, x^{\prime}\right)$ with $x^{\prime}=\left(x_{2}, \ldots, x_{n}\right)$ and use the notation

$$
D_{j}=D_{x_{j}}=\frac{\partial}{\partial x_{j}}, \quad D=\left(D_{1}, D_{2}, \ldots, D_{n}\right)=\left(D_{1}, D^{\prime}\right), \quad D^{\alpha}=D_{1}^{\alpha_{1}} \ldots D_{n}^{\alpha_{n}}
$$

for $\alpha=\left(\alpha_{1}, \ldots, \alpha_{n}\right) \in \mathbb{N}^{n}$ with $\mathbb{N}=\{0,1,2, \ldots\}$. For the sake of simplicity we always assume that the hypersurface is $N=\left\{x=\left(x_{1}, x^{\prime}\right) \in \mathbb{R}^{n} \mid x_{1}=0\right\}$. (Since the definitions and properties we shall study are of local character, we can deform any real-analytic hypersurface to a hyperplane.) Let us consider a differential operator $P$ (we always assume that a differential operator has real (or complex) analytic functions as its coefficients). $P$ is said to be a Fuchsian partial differential operator of weight $m-k$ with respect to $N$ (around $x^{0} \in N$ ) if $0 \leq k \leq m$ and if, on a neighborhood of $x^{0}, P$ is written in the form

$$
P=P\left(x, D_{x}\right)=a(x)\left(x_{1}^{k} D_{1}^{m}+\sum_{j=1}^{m} A_{j}\left(x, D^{\prime}\right) x_{1}^{\max (0, k-j)} D_{1}^{m-j}\right),
$$

where $A_{j}\left(x, D^{\prime}\right)$ is a linear partial differential operator of order at most $j$ for $j=1, \ldots, m ; A_{j}\left(0, x^{\prime}, D^{\prime}\right)$ equals a function $a_{j}\left(x^{\prime}\right)$ for $j=1, \ldots, k ; a\left(x^{\prime}\right)$ is a realanalytic function with $a\left(x^{0}\right) \neq 0$. Then the characteristic exponents $0,1, \ldots, m-$ $k-1, \lambda_{j}\left(x^{0}\right)(j=1, \ldots, k)$ of $P$ at $x^{0}$ are defined as the roots of the indicial equation

$$
\prod_{\nu=0}^{m-1}(\lambda-\nu)+\sum_{j=1}^{k} a_{j}\left(x^{0}\right) \prod_{\nu=0}^{m-j-1}(\lambda-\nu)=0
$$

in $\lambda$. In particular we call $\lambda_{j}\left(x^{0}\right)(j=1, \ldots, k)$ the non-trivial characteristic exponents of $P$.

First we briefly review the theory of elementary solutions in formal differential operators following H. Tahara [12]. We work in the complex Euclidean space $X=$ 
$\mathbb{C}^{n}$ and put $Y=\left\{z=\left(z_{1}, z^{\prime}\right) \in X \mid z_{1}=0\right\}$. Let $\widetilde{X}=\mathbb{C}^{n} \times \mathbb{C}^{n-1} \ni\left(z, w^{\prime}\right)$ with $z=\left(z_{1}, z^{\prime}\right)=\left(z_{1}, z_{2}, \ldots, z_{n}\right)$ and $w^{\prime}=\left(w_{2}, \ldots, w_{n}\right)$. Let $\widetilde{Y}$ be the hypersurface of $\widetilde{X}$ defined by $z_{1}=0$. Then we denote by $\mathcal{O}_{\widetilde{X}}$ the sheaf of holomorphic functions on $\widetilde{X}$, and by $\left.\mathcal{O}_{\widetilde{X}}\right|_{\widetilde{Y}}$ the sheaf theoretic restriction of $\mathcal{O}_{\widetilde{X}}$ to $\widetilde{Y}$. For $\varepsilon>0$, we put

$$
\begin{aligned}
W_{\varepsilon, j} & :=\left\{\left(0, z^{\prime}, w^{\prime}\right) \in \widetilde{Y}|| z^{\prime}|<\varepsilon,| w^{\prime} \mid<\varepsilon, z_{k} \neq w_{k} \text { if } 2 \leq k \leq n \text { and } k \neq j\right\}, \\
W_{\varepsilon} & :=\bigcap_{j=2}^{n} W_{\varepsilon, j} .
\end{aligned}
$$

Then the set of the formal differential operators at $0 \in Y$ is defined as

$$
\widetilde{\mathcal{D}}_{0}=\underset{\lim }{\longrightarrow}\left(\left.\mathcal{O}_{\widetilde{X}}\right|_{\widetilde{Y}}\left(W_{\varepsilon}\right) /\left.\sum_{j=2}^{n} \mathcal{O}_{\widetilde{X}}\right|_{\widetilde{Y}}\left(W_{\varepsilon, j}\right)\right)
$$

where the inductive limit is taken with respect to $\varepsilon$ tending to 0 . Hence a holomorphic function $K\left(z, w^{\prime}\right)$ defined on a neighborhood in $\widetilde{X}$ of

$$
\left\{\left(0, z^{\prime}, w^{\prime}\right)|| z^{\prime}|<\varepsilon,| w^{\prime} \mid<\varepsilon, z_{k} \neq w_{k}(2 \leq k \leq n)\right\}
$$

for some $\varepsilon>0$ defines a formal differential operator $\mathcal{K}=\left[K\left(z, w^{\prime}\right)\right] \in \widetilde{\mathcal{D}}_{0}$. Here $K\left(z, w^{\prime}\right)$ is called a kernel function of $\mathcal{K}$.

For any point $p=\left(0, z^{\prime 0}\right)$ of $Y$, we define $\widetilde{\mathcal{D}}_{p}$ by replacing the inequalities $\left|z^{\prime}\right|<\varepsilon$ and $\left|w^{\prime}\right|<\varepsilon$ in the definition of $W_{\varepsilon, j}$ by $\left|z^{\prime}-z^{\prime 0}\right|<\varepsilon$ and $\left|w^{\prime}-z^{\prime 0}\right|<\varepsilon$ respectively. Thus the sheaf $\widetilde{\mathcal{D}}$ on $Y$ of rings of formal differential operators is defined so that its stalk at $p$ coincides with $\widetilde{\mathcal{D}}_{p}$.

The sheaf $\widetilde{\mathcal{D}}$ operates on $\left.\mathcal{O}_{X}\right|_{Y}$ naturally. For example, if $K\left(z, w^{\prime}\right)$ is an element of $\left.\mathcal{O}_{\widetilde{X}}\right|_{\widetilde{Y}}\left(W_{\varepsilon}\right)$ and $f(z)$ is holomorphic on a neighborhood of $\left\{\left(0, z^{\prime}\right) \in Y \mid\right.$ $\left.\left|z^{\prime}\right|<\varepsilon\right\}$ in $X$, then we have

$$
\left(\left[K\left(z, w^{\prime}\right)\right] f\right)(z)=\left.\int_{\gamma(z)} K\left(z, w^{\prime}\right) f\left(z_{1}, w^{\prime}\right) d w^{\prime} \in \mathcal{O}_{X}\right|_{Y}\left(\left\{\left(0, z^{\prime}\right)|| z^{\prime} \mid<\varepsilon\right\}\right),
$$

where $\gamma(z)$ is an $(n-1)$-cycle defined by $\left\{w^{\prime} \in \mathbb{C}^{n-1}|| w_{k}-z_{k} \mid=\delta(2 \leq k \leq n)\right\}$ with a sufficiently small $\delta>0$.

For a linear partial differential operator $P=P\left(z, D_{z}\right)$ with holomorphic coefficients, the notion of Fuchsian operator is defined similarly with $N$ replaced by $Y$. In order to define the boundary values of hyperfunction solutions of Fuchsian partial differential equations, we use the following deep results obtained by H. Tahara [12].

Proposition 1 (Tahara [12, Corollary 1.2.13 and Theorem 1.2.14]). Let $P=$ $P\left(z, D_{z}\right)$ be a Fuchsian partial differential operator of weight $m-k$ with respect to $Y$ defined on a neighborhood of $0 \in X$. Assume that its characteristic exponents $\lambda_{1}(0), \ldots, \lambda_{k}(0)$ at 0 satisfy $\lambda_{i}(0) \notin \mathbb{Z}(i=1, \ldots, k)$ and $\lambda_{i}(0)-\lambda_{j}(0) \notin \mathbb{Z}$ if $i \neq j$. Then there are unique elements $\left[K_{i}\left(z, w^{\prime}\right)\right](i=0,1, \ldots, m-k-1)$ and 
$\left[L_{j}\left(z, w^{\prime}\right)\right](j=1, \ldots, k)$ of $\widetilde{\mathcal{D}}_{0}$ (formal elementary solutions) such that

$$
\begin{aligned}
P\left(z, D_{z}\right)\left[K_{i}\left(z, w^{\prime}\right)\right] & =0 & & (i=0, \ldots, m-k-1), \\
P\left(z, D_{z}\right)\left[L_{j}\left(z, w^{\prime}\right)\right] & =0 & & (j=1, \ldots, k), \\
{\left[D_{z_{1}}^{l} K_{i}\left(0, z^{\prime}, w^{\prime}\right)\right] } & =\delta_{i, l} \delta\left(z^{\prime}-w^{\prime}\right) & & (i, l=0, \ldots, m-k-1), \\
{\left[L_{j}\left(0, z^{\prime}, w^{\prime}\right)\right] } & =\delta\left(z^{\prime}-w^{\prime}\right) & & (j=0, \ldots, m-k-1),
\end{aligned}
$$

where $\delta_{i, l}$ denotes Kronecker's $\delta$, and

$$
\delta\left(z^{\prime}-w^{\prime}\right)=\left[\frac{1}{(-2 \pi \sqrt{-1})^{n-1}} \frac{1}{\left(z_{2}-w_{2}\right) \ldots\left(z_{n}-w_{n}\right)}\right] .
$$

Proposition 2 (Tahara [12, Theorems 1.3 .8 and 1.3.9]). Let $P$ be a Fuchsian partial differential operator of the form (1) satisfying the same condition as in Proposition 1. Let $K_{i}\left(z, w^{\prime}\right)$ and $L_{j}\left(z, w^{\prime}\right)$ be kernel functions of the formal elementary solutions of $P$ constructed in Proposition 1. Put

$$
\begin{aligned}
& A:=\left(\begin{array}{cccccccc}
0 & z_{1} & & & & & & \\
& \ddots & \ddots & & & & & \\
& & 0 & z_{1} & & & & \\
& & & 0 & 1 & & & \\
& & & & 1 & 1 & & \\
& & & & & \ddots & \ddots & \\
-z_{1} A_{m} & \ldots & \ldots & -z_{1} A_{k+1} & -A_{k} & \ldots & -A_{2} & -A_{1}+k
\end{array}\right), \\
& U\left(z, w^{\prime}\right):=\left(\vec{K}_{0}\left(z, w^{\prime}\right) \ldots \vec{K}_{m-k-1}\left(z, w^{\prime}\right) \vec{L}_{1}\left(z, w^{\prime}\right) \ldots \vec{L}_{k}\left(z, w^{\prime}\right)\right)
\end{aligned}
$$

with

$$
\begin{aligned}
\vec{K}_{i}\left(z, w^{\prime}\right):= & \left(\begin{array}{c}
K_{i}\left(z, w^{\prime}\right) \\
D_{z_{1}} K_{i}\left(z, w^{\prime}\right) \\
\vdots \\
D_{z_{1}}^{m-k-1} K_{i}\left(z, w^{\prime}\right) \\
z_{1} D_{z_{1}}^{m-k} K_{i}\left(z, w^{\prime}\right) \\
\vdots \\
z_{1}^{k} D_{z_{1}}^{m-1} K_{i}\left(z, w^{\prime}\right)
\end{array}\right) \quad(i=0, \ldots, m-k-1), \\
\vec{L}_{j}\left(z, w^{\prime}\right):= & \left(\begin{array}{c}
z_{1}^{m-k-1} L_{j}\left(z, w^{\prime}\right) \\
z_{1}^{m-k-2} \Lambda_{1}\left(\lambda_{j}\left(w^{\prime}\right)\right) L_{j}\left(z, w^{\prime}\right) \\
\vdots \\
\Lambda_{m-k-1}\left(\lambda_{j}\left(w^{\prime}\right)\right) L_{j}\left(z, w^{\prime}\right) \\
\Lambda_{m-k}\left(\lambda_{j}\left(w^{\prime}\right)\right) L_{j}\left(z, w^{\prime}\right) \\
\vdots \\
\Lambda_{m-1}\left(\lambda_{j}\left(w^{\prime}\right)\right) L_{j}\left(z, w^{\prime}\right)
\end{array}\right) \quad(j=1, \ldots, k)
\end{aligned}
$$


where $\lambda_{1}\left(z^{\prime}\right), \ldots, \lambda_{k}\left(z^{\prime}\right)$ are the non-trivial characteristic exponents of $P$, and

$$
\begin{aligned}
& \Lambda_{i}\left(\lambda_{j}\left(w^{\prime}\right)\right) \\
& \quad:=\left(z_{1} D_{z_{1}}+\lambda_{j}\left(w^{\prime}\right)\right)\left(z_{1} D_{z_{1}}+\lambda_{j}\left(w^{\prime}\right)-1\right) \ldots\left(z_{1} D_{z_{1}}+\lambda_{j}\left(w^{\prime}\right)-i+1\right) .
\end{aligned}
$$

Then the differential equation $P u=0$ is equivalent to the system of differential equations $\left(z_{1} D_{z_{1}}-A\right) \vec{u}=0$ by the correspondence

$$
\vec{u}=\left(\begin{array}{c}
u \\
D_{z_{1}} u \\
\vdots \\
D_{z_{1}}^{m-k-1} u \\
z_{1} D_{z_{1}}^{m-k} u \\
\vdots \\
z_{1}^{k} D_{z_{1}}^{m-1} u
\end{array}\right)
$$

and $\mathcal{U}:=\left[U\left(z, w^{\prime}\right)\right]$ is an invertible matrix with elements in $\widetilde{\mathcal{D}}_{0}$ satisfying $\mathcal{U}^{-1}\left(z_{1} D_{z_{1}}-A\left(z, D_{z}\right)\right) \mathcal{U}$

$$
=z_{1} D_{z_{1}}-\left(\begin{array}{ccccc}
0 & & & & \\
& \ddots & & & \\
& & 0 & & \\
& & \lambda_{1}\left(z^{\prime}\right)-m+k+1 & & \\
& & & \ddots & \\
& & & & \lambda_{k}\left(z^{\prime}\right)-m+k+1
\end{array}\right) \text {. }
$$

ExAmPle (the Euler-Poisson-Darboux equation). Put $n=2$ and

$$
P=z_{1}\left(D_{z_{1}}^{2}-D_{z_{2}}^{2}\right)+a D_{z_{1}}+b D_{z_{2}}
$$

with constants $a, b \in \mathbb{C}$. Then $P$ is a Fuchsian operator of weight 1 and the non-trivial characteristic exponent is $1-a$. Assume $a \notin \mathbb{Z}$. Then the formal elementary solutions of $P$ are given by

$$
\begin{aligned}
& K_{0}\left(z_{1}, z_{2}, w_{2}\right)=\frac{-1}{2 \pi \sqrt{-1}} \frac{1}{z_{1}+z_{2}-w_{2}} F\left(1, \frac{a+b}{2}, a ; \frac{2 z_{1}}{z_{1}+z_{2}-w_{2}}\right), \\
& L_{1}\left(z_{1}, z_{2}, w_{2}\right)=\frac{-1}{2 \pi \sqrt{-1}} \frac{1}{z_{1}+z_{2}-w_{2}} F\left(1,1-\frac{a-b}{2}, 2-a ; \frac{2 z_{1}}{z_{1}+z_{2}-w_{2}}\right),
\end{aligned}
$$

where $F$ denotes the Gauss hypergeometric function. We remark that in this case $F$ can be explicitly given by

$$
F(1, \beta, \gamma ; z)=(\gamma-1) z^{1-\gamma}(1-z)^{\gamma-\beta-1} \int_{0}^{z} \tau^{\gamma-2}(1-\tau)^{\beta-\gamma} d \tau
$$

for $|z|<1$ if $\operatorname{Re}(\gamma)>1$. 
1.2. Boundary values of hyperfunction solutions to Fuchsian partial differential equations. We use the notation of 1.1. Put $M_{+}=\left\{x \in M \mid x_{1}>0\right\}$. Let $\mathcal{B}_{M}$ be the sheaf of hyperfunctions on $M$. We define the sheaf $\mathcal{B}_{N \mid M_{+}}$on $N$ so that its stalk at $p=\left(0, x^{\prime 0}\right) \in N$ is

$$
\left(\mathcal{B}_{N \mid M_{+}}\right)_{p}=\underset{\lim }{\longrightarrow} \mathcal{B}_{M}\left(\left\{x \in M_{+}|| x-p \mid<\varepsilon\right\}\right),
$$

where the inductive limit is taken with respect to $\varepsilon>0$ tending to 0 .

In [7], we constructed a sheaf $\widetilde{\mathcal{B}}_{N \mid M_{+}}$on $N$ containing $\mathcal{B}_{N \mid M_{+}}$as a subsheaf such that the sheaf $\widetilde{\mathcal{D}}$ of formal differential operators operates on $\widetilde{\mathcal{B}}_{N \mid M_{+}}$.

Let $P$ be a Fuchsian partial differential operator of weight $m-k$ with respect to $N$ defined on a neighborhood of $x^{0} \in N$. Assume that its characteristic exponents $\lambda_{1}\left(x^{0}\right), \ldots, \lambda_{k}\left(x^{0}\right)$ at $x^{0}$ satisfy $\lambda_{i}\left(x^{0}\right) \notin \mathbb{Z}$ and $\lambda_{i}\left(x^{0}\right)-\lambda_{j}\left(x^{0}\right) \notin \mathbb{Z}$ if $i \neq j$. Let $\mathcal{K}_{i}=\left[K_{i}\left(z, w^{\prime}\right)\right]$ and $\mathcal{L}_{j}=\left[L_{j}\left(z, w^{\prime}\right)\right]$ be the formal elementary solutions of $P$. Then by Proposition 2, any element $u(x)$ of $\widetilde{\mathcal{B}}_{N \mid M_{+}}$satisfying $P u=0$ is written in the form

$$
u(x)=\sum_{i=0}^{m-k-1} \mathcal{K}_{i} u_{i}\left(x^{\prime}\right)+\sum_{j=1}^{k} \mathcal{L}_{j}\left(x_{1}^{\lambda_{j}\left(x^{\prime}\right)} v_{j}\left(x^{\prime}\right)\right)
$$

as an identity in $\widetilde{\mathcal{B}}_{N \mid M_{+}}$with unique hyperfunctions

$$
u_{0}\left(x^{\prime}\right), \ldots, u_{m-k-1}\left(x^{\prime}\right), \quad v_{1}\left(x^{\prime}\right), \ldots, v_{k}\left(x^{\prime}\right)
$$

on a neighborhood of $x_{0}$ in $N$. In particular, for a hyperfunction solution $u(x) \in$ $\mathcal{B}_{N \mid M_{+}}$of $P u=0$, the regular and the singular boundary values

$$
\gamma_{+\operatorname{reg}}(u)=\left(\begin{array}{c}
u_{0}\left(x^{\prime}\right) \\
\vdots \\
u_{m-k-1}\left(x^{\prime}\right)
\end{array}\right) \in\left(\mathcal{B}_{N}\right)^{m-k}, \quad \gamma_{+\operatorname{sing}}(u)=\left(\begin{array}{c}
v_{1}\left(x^{\prime}\right) \\
\vdots \\
v_{k}\left(x^{\prime}\right)
\end{array}\right) \in\left(\mathcal{B}_{N}\right)^{k}
$$

are defined as above. We call $\gamma_{+ \text {reg }}(u)$ and $\gamma_{+\operatorname{sing}}(u)$ the boundary values of $u(x)$.

2. Main results on reflection of singularities. We use the same notation as in the preceding section and define the purely imaginary cosphere bundles of $M$ and $N$ by

$$
\begin{aligned}
& \sqrt{-1} S^{*} M=M \times \sqrt{-1} S^{n-1}=\left\{(x, \sqrt{-1}\langle\xi, d x\rangle \infty) \mid x \in M, \xi \in \mathbb{R}^{n} \backslash\{0\}\right\}, \\
& \sqrt{-1} S^{*} N=N \times \sqrt{-1} S^{n-2}=\left\{\left(0, x^{\prime}, \sqrt{-1}\left\langle\xi^{\prime}, d x^{\prime}\right\rangle \infty\right) \mid x^{\prime}, \xi^{\prime} \in \mathbb{R}^{n-1} \backslash\{0\}\right\},
\end{aligned}
$$

where $\langle\xi, d x\rangle=\sum_{j=1}^{n} \xi_{j} d x_{j}$, and the symbol $\infty$ denotes taking cosets in $S^{n-1}=$ $\mathbb{R}^{n} / \mathbb{R}_{+}$with $\mathbb{R}_{+}$being the set of positive real numbers.

We consider a linear partial differential operator $P$ with analytic coefficients of the form

$$
P(x, D)=x_{1} P_{m}(x, D)+Q(x, D)
$$

defined on a neighborhood (in $M$ ) of a point $x^{0}$ of $N$. We assume 
(A-1) $\quad P_{m}(x, D)=\sum_{|\alpha| \leq m} a_{\alpha}(x) D^{\alpha}$ is a linear partial differential operator of order $m$ and $N$ is non-characteristic for $P$ at $x^{0}$ (i.e. $a_{(m, 0, \ldots, 0)}\left(x^{0}\right) \neq 0$ ).

(A-2) $\quad Q(x, D)=\sum_{|\alpha| \leq m-1} b_{\alpha}(x) D^{\alpha}$ is a linear partial differential operator of order $m-1$.

Then $P$ is a Fuchsian linear partial differential operator of weight $m-1$ with respect to $N$ in the sense of Baouendi-Goulaouic [1]. The non-trivial characteristic exponent of $P$ at $x^{0}$ is

$$
\lambda\left(x^{0}\right)=m-1-\frac{b_{(m-1,0, \ldots, 0)}\left(x^{0}\right)}{a_{(m, 0, \ldots, 0)}\left(x^{0}\right)} .
$$

We assume

(A-3) $\quad \lambda\left(x^{0}\right) \notin \mathbb{Z}$.

Let $u(x)$ be a hyperfunction on $U \cap M_{+}$satisfying $P u(x)=0$ with a sufficiently small open neighborhood $U$ of $x^{0}$ in $M$. Then the boundary values of $u(x)$

$$
\gamma_{+\mathrm{reg}}(u) \in \mathcal{B}_{N}(U \cap N)^{m-1}, \quad \gamma_{+\operatorname{sing}}(u) \in \mathcal{B}_{N}(U \cap N)
$$

have been defined in the preceding section. Put

$$
p_{m}(x, \xi)=\sigma_{m}(P)(x, \xi)=\sum_{|\alpha|=m} a_{\alpha}(x) \xi^{\alpha}, \quad q_{m-1}(x, \xi)=\sum_{|\alpha|=m-1} b_{\alpha}(x) \xi^{\alpha}
$$

and let $x^{*}=\left(x^{0}, \sqrt{-1}\left\langle\xi^{\prime 0}, d x^{\prime}\right\rangle \infty\right)$ be a point of $\sqrt{-1} S^{*} N$. Assume

(A-4) $\quad P_{m}$ is microlocally strictly hyperbolic at $x^{*}$; i.e., the roots of the equation $p_{m}\left(x, \zeta, \xi^{\prime}\right)=0$ in $\zeta$ are all real and distinct if $x \in M$ with $\left|x-x^{0}\right|<\varepsilon$ and $\xi^{\prime} \in \mathbb{R}^{n-1}$ with $\left|\xi^{\prime}-\xi^{\prime 0}\right|<\varepsilon$ for some $\varepsilon>0$.

Then there are $m$ bicharacteristics $b_{j}\left(x^{*}\right)(j=1, \ldots, m)$ of $P_{m}$ issuing from

$$
x^{*(j)}=\left.\left(x^{0}, \sqrt{-1}\left(\xi_{1}^{(j)} d x_{1}+\left\langle\xi^{\prime 0}, d x^{\prime}\right\rangle\right) \infty\right) \in \sqrt{-1} S^{*} M\right|_{N},
$$

where $\zeta=\xi_{1}^{(j)}(j=1, \ldots, m)$ are the roots of $p_{m}\left(x^{0}, \zeta, \xi^{\prime 0}\right)=0$. (We may assume $\xi_{1}^{(0)}<\xi_{1}^{(1)}<\ldots<\xi_{1}^{(m)}$.) We denote by $b_{j}^{+}\left(x^{*}\right)$ the part of $b_{j}\left(x^{*}\right)$ where $x_{1}>0$. We define a function $e$ on $\sqrt{-1} S^{*} M$ by

$$
e((x, \sqrt{-1}\langle\xi, d x\rangle \infty))=-\frac{q_{m-1}(x, \xi)}{\left(\partial / \partial \xi_{1}\right) p_{m}(x, \xi)} .
$$

Example. Put

$$
P=x_{1}\left(D_{1}^{2}+\ldots+D_{k}^{2}-D_{k+1}^{2}-\ldots-D_{n}^{2}\right)+\sum_{j=1}^{n} a_{j}(x) D_{j}+b(x)
$$

with $1 \leq k \leq n-1$ and real-analytic functions $a_{j}(x)$ and $b(x)$ defined on a neighborhood of $x^{0} \in N$ such that $a_{1}\left(x^{0}\right) \notin \mathbb{Z}$. Then $P$ satisfies (A-1)-(A-4) for $x^{*}=\left(x^{0}, \sqrt{-1}\left\langle\xi^{\prime 0}, d x\right\rangle \infty\right) \in \sqrt{-1} S^{*} N$ if $\xi^{\prime 0}=\left(\xi_{2}^{0}, \ldots, \xi_{n}^{0}\right)$ satisfies

$$
\left(\xi_{2}^{0}\right)^{2}+\ldots+\left(\xi_{k}^{0}\right)^{2}<\left(\xi_{k+1}^{0}\right)^{2}+\ldots+\left(\xi_{n}^{0}\right)^{2} .
$$


For a hyperfunction $u$ defined on an open subset of $M$, we denote by $\operatorname{SS}(u)$ the singularity spectrum (= analytic wave front set) of $u$, which is a subset of $\sqrt{-1} S^{*} M$.

THEOREM 1. Let $u(x)$ be a hyperfunction on $U \cap M_{+}$with an open neighborhood $U$ of $x^{0}$ in $M$. Let $k$ be an arbitrary integer with $1 \leq k \leq m$ and assume (in addition to $(\mathrm{A}-1)-(\mathrm{A}-4))$

- $P u(x)=0$,

- $\gamma_{+\operatorname{reg}}(u)$ is micro-analytic at $x^{*}$,

- $\operatorname{SS}(u) \cap b_{k}^{+}\left(x^{*}\right)=\emptyset$,

- $e\left(x^{*(k)}\right) \notin\{-1,-2,-3, \ldots\}$.

Then $\operatorname{SS}(u) \cap b_{j}^{+}\left(x^{*}\right)=\emptyset$ for any $j$ and $\gamma_{+\operatorname{sing}}(u)$ is micro-analytic at $x^{*}$.

Rem ark 1. The last assumption in Theorem 1 is necessary as is seen in the following example: Put

$$
P=x_{1}\left(D_{1}^{2}-D_{2}^{2}\right)+a D_{1}+(2-a) D_{2}
$$

in $\mathbb{R}^{2}$ with $a \in \mathbb{C} \backslash \mathbb{Z}$ and put $x^{*}=\left(0, \sqrt{-1} d x_{2} \infty\right)$. Then we have $\lambda=1-a$ and

$$
\begin{gathered}
x^{*(1)}=\left(0, \sqrt{-1}\left(d x_{1}+d x_{2}\right) \infty\right), \quad x^{*(2)}=\left(0, \sqrt{-1}\left(-d x_{1}+d x_{2}\right) \infty\right), \\
b_{1}^{+}\left(x^{*}\right)=\left\{\left(x_{1}, x_{2}, \sqrt{-1}\left(d x_{1}+d x_{2}\right) \infty\right) \mid x_{1}>0, x_{2}=-x_{1}\right\}, \\
b_{2}^{+}\left(x^{*}\right)=\left\{\left(x_{1}, x_{2}, \sqrt{-1}\left(-d x_{1}+d x_{2}\right) \infty\right) \mid x_{1}>0, x_{2}=x_{1}\right\}, \\
e\left(x^{*(1)}\right)=-1, \quad e\left(x^{*(2)}\right)=1-a .
\end{gathered}
$$

Define a hyperfunction $u$ on $M_{+}$by

$$
u\left(x_{1}, x_{2}\right)=x_{1}^{1-a}\left(x_{2}+\sqrt{-1} 0-x_{1}\right)^{a-1} .
$$

Then $u$ satisfies $P u=0$ and

$$
\gamma_{+\operatorname{reg}}(u)=0, \quad \gamma_{+\operatorname{sing}}(u)=\left(x_{2}+\sqrt{-1} 0\right)^{a-1}, \quad \operatorname{SS}(u)=b_{2}^{+}\left(x^{*}\right) .
$$

THEOREM 2. Let $u(x)$ be a hyperfunction on $U \cap M_{+}$with an open neighborhood $U$ of $x^{0}$ in $M$. Let $k$ be an arbitrary integer with $1 \leq k \leq m$ and assume (in addition to (A-1)-(A-4))

- $P u(x)=0$,

- $\gamma_{+\operatorname{sing}}(u)$ is micro-analytic at $x^{*}$,

- $\operatorname{SS}(u) \cap b_{j}^{+}\left(x^{*}\right)=\emptyset$ for any $j \neq k$,

- $e\left(x^{*(k)}\right) \notin\{0,1,2, \ldots\}$.

Then $\operatorname{SS}(u) \cap b_{k}^{+}\left(x^{*}\right)=\emptyset$ and $\gamma_{+\operatorname{reg}}(u)$ is micro-analytic at $x^{*}$.

Remark 2. The last assumption in Theorem 2 is necessary as is seen in the following example: Put

$$
P=x_{1}\left(D_{1}^{2}-D_{2}^{2}\right)+a D_{1}+a D_{2}
$$


in $\mathbb{R}^{2}$ with $a \in \mathbb{C} \backslash \mathbb{Z}$ and put $x^{*}=\left(0, \sqrt{-1} d x_{2} \infty\right)$. Then we have $\lambda=1-a$ and

$$
\begin{gathered}
x^{*(1)}=\left(0, \sqrt{-1}\left(d x_{1}+d x_{2}\right) \infty\right), \quad x^{*(2)}=\left(0, \sqrt{-1}\left(-d x_{1}+d x_{2}\right) \infty\right), \\
b_{1}^{+}\left(x^{*}\right)=\left\{\left(x_{1}, x_{2}, \sqrt{-1}\left(d x_{1}+d x_{2}\right) \infty\right) \mid x_{1}>0, x_{2}=-x_{1}\right\}, \\
b_{2}^{+}\left(x^{*}\right)=\left\{\left(x_{1}, x_{2}, \sqrt{-1}\left(-d x_{1}+d x_{2}\right) \infty \mid x_{1}>0, x_{2}=x_{1}\right\},\right. \\
e\left(x^{*(1)}\right)=-a, \quad e\left(x^{*(2)}\right)=0 .
\end{gathered}
$$

Define a hyperfunction $u$ on $M_{+}$by

$$
u\left(x_{1}, x_{2}\right)=\left(x_{2}+\sqrt{-1} 0-x_{1}\right)^{1-a} .
$$

Then $u$ satisfies $P u=0$ and

$$
\gamma_{+\operatorname{reg}}(u)=\left(x_{2}+\sqrt{-1} 0\right)^{1-a}, \quad \gamma_{+\operatorname{sing}}(u)=0, \quad \operatorname{SS}(u)=b_{2}^{+}\left(x^{*}\right) .
$$

As an application of these theorems, we give results for rather special equations. The first is concerning a non-characteristic boundary value problem with two bicharacteristics issuing from the same point:

TheOREm 3. Let $P$ be a linear partial differential operator of the form

$$
P=D_{1}^{2}-x_{1}^{k} A\left(x^{\prime}, D^{\prime}\right),
$$

with $k \geq 0$ an integer, defined on a neighborhood of $x^{0} \in N$, where $A$ is a second order linear partial differential operator free of $x_{1}$ and $D_{1}$ such that $\sigma_{2}(A)\left(x^{\prime}, \xi^{\prime}\right)$ is real-valued for real $x^{\prime}, \xi^{\prime}$ and that $\sigma_{2}(A)\left(x^{0}, \xi^{\prime 0}\right)>0$. Let $b_{1}^{+}, b_{2}^{+}$be the two bicharacteristics in $\left.\sqrt{-1} S^{*} M\right|_{M_{+}}$of $P$ issuing from $x^{*}=\left(x^{0}, \sqrt{-1}\left\langle\xi^{\prime 0}, d x^{\prime}\right\rangle \infty\right)$. Let $u(x)$ be a hyperfunction on $U \cap M_{+}$with an open neighborhood $U$ of $x^{0}$ such that

- $P u(x)=0$,

- $\left.u\right|_{x_{1} \rightarrow+0}$ or $\left.D_{1} u\right|_{x_{1} \rightarrow+0}$ is micro-analytic at $x^{*}$,

- $\operatorname{SS}(u) \cap b_{1}^{+}=\emptyset$.

Then $\operatorname{SS}(u) \cap b_{2}^{+}=\emptyset$, and both $\left.u\right|_{x_{1} \rightarrow+0}$ and $\left.D_{1} u\right|_{x_{1} \rightarrow+0}$ are micro-analytic at $x^{*}$.

The last result is for a Fuchsian partial differential equation with two bicharacteristics issuing from the same point $(k \geq 2)$ or both tangent to the boundary $(k=0)$.

TheOREM 4. Let $P$ be a linear partial differential operator of the form

$$
P=x_{1} D_{1}^{2}-x_{1}^{k} A\left(x^{\prime}, D^{\prime}\right)+a_{1}\left(x^{\prime}\right) D_{1},
$$

with an integer $k \geq 2$ or $k=0$ defined on a neighborhood of $x^{0} \in N$, where $A$ is a second order linear partial differential operator satisfying the same conditions as in Theorem 3 , and $a_{1}\left(x^{\prime}\right)$ is a real-analytic function defined on a neighborhood of $x^{0}$ with $a_{1}\left(x^{0}\right) \notin \mathbb{Z}$ and $2\left(1-a_{1}\left(x^{0}\right)\right) /(k+1) \notin \mathbb{Z}$. Let $b_{1}^{+}, b_{2}^{+}$be the two bicharacteristics in $\left.\sqrt{-1} S^{*} M\right|_{M_{+}}$of $P$ issuing from $\left(x^{0}, \sqrt{-1}\left\langle\xi^{\prime 0}, d x^{\prime}\right\rangle \infty\right)$ (when $k \geq 2)$ or issuing from $\left(x^{0}, \sqrt{-1} d x_{1} \infty\right)$ (when $\left.k=0\right)$. Let $u(x)$ be a hyperfunction on $U \cap M_{+}$with $U$ an open neighborhood of $x^{0}$ such that 
- $P u(x)=0$,

- $\gamma_{+\operatorname{reg}}(u)$ or $\gamma_{+\operatorname{sing}}(u)$ is micro-analytic at $x^{*}$,

- $\operatorname{SS}(u) \cap b_{1}^{+}=\emptyset$.

Then $\operatorname{SS}(u) \cap b_{2}^{+}=\emptyset$, and both $\gamma_{+\operatorname{reg}}(u)$ and $\gamma_{+\operatorname{sing}}(u)$ are micro-analytic at $x^{*}$.

\section{Proof of the main results}

Proof of Theorem 1. There exists a hyperfunction $\widetilde{u}$ on $U$ such that $\widetilde{u}=u$ in $U \cap M_{+}, \widetilde{u}=0$ in $U \cap M_{-}$and that

$$
P \widetilde{u}(x)=\sum_{\mu=0}^{m-2} \delta^{(\mu)}\left(x_{1}\right) \sum_{\nu=0}^{m-2-\mu} B_{\mu, \nu}\left(x^{\prime}, D^{\prime}\right) \gamma_{+\operatorname{reg}}(u)_{\nu},
$$

where $\gamma_{+ \text {reg }}(u)_{\nu}$ denotes the $(\nu-1)$ th component of $\gamma_{+\operatorname{reg}}(u)$, and $B_{\mu, \nu}\left(x^{\prime}, D^{\prime}\right)$ is a linear partial differential operator (cf. [12, Proposition 2.3.10]). Hence $P \widetilde{u}(x)$ is micro-analytic on a neighborhood of

$$
\left\{\left(x^{0}, \sqrt{-1}\left(\xi_{1} d x_{1}+\left\langle\xi^{\prime}, d x^{\prime}\right\rangle\right) \infty\right) \in \sqrt{-1} S^{*} M \mid \xi_{1} \in \mathbb{R}, \xi^{\prime}=\xi^{\prime 0}\right\} .
$$

The assumption (A-4) implies that $P_{m}$ is of real simple characteristics near $x^{*(j)}$ 's. Since $e\left(x^{*(k)}\right)$ is not a negative integer, we can apply the theorem on branching of singularities by Ôaku [6, Theorem 3.5] and get $x^{*(k)} \notin \mathrm{SS}(\widetilde{u})$. (Note that $\mathrm{SS}(\widetilde{u}) \subset\left\{x_{1} \geq 0\right\}$.) Hence we have

$\mathrm{SS}(\widetilde{u}) \cap\left\{\left(x^{0}, \sqrt{-1}\left(\xi_{1} d x_{1}+\left\langle\xi^{\prime 0}, d x^{\prime}\right\rangle\right) \infty\right) \in \sqrt{-1} S^{*} M \mid \xi_{1}^{(k-1)}<\xi_{1}<\xi_{1}^{(k+1)}\right\}=\emptyset$, where we put $\xi_{1}^{(0)}=-\infty, \xi_{1}^{(m+1)}=\infty$. Since $P_{m}$ is micro-hyperbolic in the direction $d x_{1}+d \xi_{1}$, we get

$$
x^{*(j)} \notin \mathrm{SS}(\widetilde{u})
$$

for $j=k-1$ and $k+1$. Hence we get (2) for any $j$ by induction. This implies $\mathrm{SS}(u) \cap b_{j}^{+}\left(x^{*}\right)=\emptyset$ for any $j$. The theorem on the propagation of micro-analyticity up to the boundary by Ôaku [8, Theorem 2.2] implies that $\gamma_{+\operatorname{sing}}(u)$ is microanalytic at $x^{*}$. This completes the proof of Theorem 1 .

Proof of Theorem 2. By (A-4) and [5, Theorem 2] there exists a hyperfunction $\widetilde{u}(x)$ on a neighborhood $V \subset U$ of $x^{0}$ with $x_{1}$ as a real-analytic parameter such that $P \widetilde{u}=0$ and that $\gamma_{+\operatorname{reg}}(\widetilde{u}-u)_{\nu}=\left.D_{1}^{\nu} \widetilde{u}\right|_{x_{1}=0}-\gamma_{+\operatorname{reg}}(u)_{\nu}$ is micro-analytic at $x^{*}$ for any $\nu=0, \ldots, m-2$. Moreover, $\gamma_{+\operatorname{sing}}(\widetilde{u}-u)=-\gamma_{+\operatorname{sing}}(u)$ is microanalytic at $x^{*}$ by the assumption. Then by the micro-local uniqueness theorem for Fuchsian boundary value problems [8, Theorem 2.1] we have

$$
\operatorname{SS}(u-\widetilde{u}) \cap\left\{(x, \sqrt{-1}\langle\xi, d x\rangle \infty)|| x\left|<\varepsilon, x_{1}>0,\right| \xi^{\prime}-\xi^{\prime 0} \mid<\varepsilon\right\}=\emptyset,
$$

namely, $\operatorname{SS}(\widetilde{u})=\mathrm{SS}(u)$ holds on

$$
\left\{(x, \sqrt{-1}\langle\xi, d x\rangle \infty)|| x\left|<\varepsilon, x_{1}>0,\right| \xi^{\prime}-\xi^{\prime 0} \mid<\varepsilon\right\}
$$


for some $\varepsilon>0$. Since $\widetilde{u}$ has $x_{1}$ as a real-analytic parameter it follows that

$\mathrm{SS}(\widetilde{u}) \cap\left\{\left(x^{0}, \sqrt{-1}\left(\xi_{1} d x_{1}+\left\langle\xi^{\prime 0}, d x^{\prime}\right\rangle\right) \infty\right) \in \sqrt{-1} S^{*} M \mid \xi_{1}<\xi_{1}^{(1)}\right.$ or $\left.\xi_{1}>\xi_{1}^{(m)}\right\}=\emptyset$.

Since $P$ is micro-hyperbolic in the direction $d x_{1}+d \xi_{1}$, (2) holds for $j=1$ and $j=m$ if $j \neq k$. Hence we get

$$
\operatorname{SS}(\widetilde{u}) \cap\left\{\left(x^{0}, \sqrt{-1}\left(\xi_{1} d x_{1}+\left\langle\xi^{\prime 0}, d x^{\prime}\right\rangle\right) \infty\right) \in \sqrt{-1} S^{*} M \mid \xi_{1} \neq \xi_{1}^{(k)}\right\}=\emptyset .
$$

Again by [6, Theorem 3.5] and the condition that $e\left(x^{*(k)}\right) \notin\{0,1,2, \ldots\}$ we get $(2)$ for $j=k$ and hence $\operatorname{SS}(u) \cap b_{k}^{+}\left(x^{*}\right)=\emptyset$. This completes the proof of Theorem 2 .

Proof of Theorem 3. We use a local coordinate transformation $x_{1}=t^{\alpha}$ with $\alpha=2 /(k+2)$. We define a hyperfunction $v\left(t, x^{\prime}\right)$ by $v\left(t, x^{\prime}\right)=u\left(t^{\alpha}, x^{\prime}\right)$ for $t>0$. Then $v\left(t, x^{\prime}\right)$ satisfies $Q v\left(t, x^{\prime}\right)=0$ with

$$
Q=t D_{t}^{2}+(1-\alpha) D_{t}-\alpha^{2} t A\left(x^{\prime}, D^{\prime}\right) .
$$

This $Q$ satisfies (A-1)-(A-4). In fact, for $Q$ we have

$$
\lambda=\alpha \notin \mathbb{Z}, \quad e\left(x^{*(j)}\right)=\alpha-1 \notin \mathbb{Z} \quad \text { for } j=1,2 .
$$

Moreover, we can show

$$
u\left(+0, x^{\prime}\right)=\gamma_{+\operatorname{reg}}(v)\left(x^{\prime}\right), \quad D_{1} u\left(+0, x^{\prime}\right)=\gamma_{+\operatorname{sing}}(v)\left(x^{\prime}\right) .
$$

Hence Theorem 3 follows from Theorems 1 and 2 .

Proof of Theorem 4. We use a local coordinate transformation $x_{1}=t^{\alpha}$ with $\alpha=2 /(k+1)$. We define a hyperfunction $v\left(t, x^{\prime}\right)$ by $v\left(t, x^{\prime}\right)=u\left(t^{\alpha}, x^{\prime}\right)$ for $t>0$. Then $v\left(t, x^{\prime}\right)$ satisfies $Q v\left(t, x^{\prime}\right)=0$ with

$$
Q=t D_{t}^{2}+\left(1+\alpha-\alpha a_{1}\left(x^{\prime}\right)\right) D_{t}-\alpha^{2} t A\left(x^{\prime}, D^{\prime}\right) .
$$

This $Q$ satisfies (A-1)-(A-4). In fact, for $Q$ we have

$$
\begin{gathered}
\lambda=\alpha\left(a_{1}\left(x^{0}\right)-1\right) \notin \mathbb{Z}, \\
e\left(x^{*(j)}\right)=\left(1+\alpha-\alpha a_{1}\left(x^{\prime}\right)\right) / 2 \notin \mathbb{Z} \quad \text { for } j=1,2 .
\end{gathered}
$$

Moreover, we can show

$$
\gamma_{+\operatorname{reg}}(u)\left(x^{\prime}\right)=\gamma_{+\operatorname{reg}}(v)\left(x^{\prime}\right), \quad \gamma_{+\operatorname{sing}}(u)\left(x^{\prime}\right)=\gamma_{+\operatorname{sing}}(v)\left(x^{\prime}\right) .
$$

Hence Theorem 4 follows from Theorems 1 and 2 .

\section{References}

[1] M. S. Baouendi and C. Goulaouic, Cauchy problems with characteristic initial hypersurface, Comm. Pure Appl. Math. 26 (1973), 455-475.

[2] M. Kashiwara and T. Oshima, Systems of differential equations with regular singularities and their boundary value problems, Ann. of Math. 106 (1977), 145-200.

[3] Y. Laurent et T. Monteiro Fernandes, Systèmes différentiels Fuchsiens le long d'une sous-variété, Publ. RIMS Kyoto Univ. 24 (1988), 397-431.

[4] L. Nirenberg, Lectures on Linear Partial Differential Equations, Regional Conf. Ser. in Math. 17, Amer. Math. Soc., 1973. 
[5] T. Ôaku, Micro-local Cauchy problems and local boundary value problems, Proc. Japan Acad. 55 (1979), 136-140.

[6] -, A canonical form of a system of microdifferential equations with non-involutory characteristics and branching of singularities, Invent. Math. 65 (1982), 491-525.

[7] - Boundary value problems for systems of linear partial differential equations and propagation of micro-analyticity, J. Fac. Sci. Univ. Tokyo 33 (1986), 175-232.

[8] -, Removable singularities of solutions of linear partial differential equations, ibid., 403428.

[9] T. Oshima, A definition of boundary values of solutions of partial differential equations with regular singularities, Publ. RIMS Kyoto Univ. 19 (1983), 1203-1230.

[10] -, Boundary value problems for systems of linear partial differential equations with regular singularities, Advanced Studies in Pure Math. 4 (1984), 391-432.

[11] P. Schapira, Propagation at the boundary and reflection of analytic singularities of solutions of linear partial differential equations I, Publ. RIMS Kyoto Univ. 12 Suppl. (1977), 441-453.

[12] H. Tahara, Fuchsian type equations and Fuchsian hyperbolic equations, Japan. J. Math. 5 (1979), 245-347. 